\title{
LÍNEA BASE DE CONOCIMIENTO EN ESTABLECIMIENTO Y MANEJO DE PLANTACIONES FORESTALES DE PEQUEÑOS PROPIETARIOS DE LA PROVINCIA DE ARAUCO REGIÓN DEL BIO BIO
}

Aguilera, Mauricio; García, Edison y Villarroel, Arnoldo.

\section{RESUMEN}

Existe consenso a nivel nacional de la existencia de brechas tecnológicas en la producción forestal entre las grandes empresas del sector y los pequeños y medianos propietarios. Los motivos se asocian a los niveles económicos, acceso a conocimiento técnico y gestión de la producción.

En este estudio se muestra un análisis de línea base de conocimientos que tienen pequeños propietarios en el tema del establecimiento y manejo de plantaciones forestales, de las Comunas de Cañete, Lebu y Los Álamos, Provincia de Arauco, Región del Bio Bio.

Los datos fueron recopilados a través de encuestas semiestructuradas y visitas a terreno. El análisis de los antecedentes revela que los pequeños propietarios tienen un bajo conocimiento de la técnica de establecimiento de plantaciones y un muy bajo conocimiento sobre el manejo de estas.

Palabras clave: Plantaciones forestales, Pequeños y medianos propietarios.

\section{SUMMARY}

Well known are the technical gaps in forest production between small and medium owners and the big forests companies, and these gaps are associated to the economical levels, the access to technical knowledge and the production management.

The present study shows a small and medium owners base line analysis on their knowledge regarding to planted forests establishment and management in the Cañete, Lebu and Los Álamos, Communes, Arauco Province, Bio Bio Region.

The collected data, through a semi structured survey and field visits, revealed a small and medium owner's scarce knowledge on plantation establishment techniques and a very short acquired knowledge on planted forests management.

Keywords: Planted forests, Small and medium owners.

${ }^{1}$ Instituto Forestal, Sede Bio Bio, Chile. maguilera@infor.cl; egarcía@infor.cl; avillarr@infor.cl 


\section{INTRODUCCIÓN}

El sector forestal chileno se caracteriza por contribuir significativamente a la economía nacional, en el año 2018 genera retornos de exportaciones por US\$ FOB 6.838 millones (INFOR, 2018). Sin embargo, siendo un sector consolidado y exitoso en el segmento de exportación y producción industrial asociado a las grandes empresas, no lo es para el segmento de pequeños y medianos empresarios y propietarios de suelos y bosques, dado que falta desarrollar integralmente toda la cadena productiva y sus componentes en estos segmentos de propietarios.

En Chile operan tres grandes empresas forestales cuya capacidad económica les permite adoptar con relativa facilidad los cambios y oportunidades tecnológicas, mientras que, en el otro extremo, a la pequeña empresa se le hace difícil innovar e incorporar tecnología que mejore la productividad de sus cultivos y la rentabilidad de sus operaciones.

La superficie de plantaciones forestales constituye la base de recursos sobre los cuales se ha forjado el crecimiento de la industria forestal, estas plantaciones principalmente de pino (Pinus radiata) y eucalipto (Eucalyptus globulus y E. nitens) han aumentado sostenidamente en los últimos 40 años permitiendo un nivel de existencias que ha permitido la consolidación de la industria forestal.

En el periodo 1978 -2016 se triplicó la superficie de plantaciones forestales en Chile, pasando de 711 mil hectáreas a 2,4 millones de hectáreas, periodo en el cual se mantuvo en vigencia la legislación de fomento forestal que entregaba incentivos estatales a la forestación (DL 701 de 1974). Actualmente, las especies principales que componen estas plantaciones son pino radiata $(57,6 \%)$, Eucalyptus globulus $(24,5 \%)$ y E. nitens $(11,1 \%)$ (INFOR, 2018).

Respecto de la propiedad de estos bosques plantados, las tres grandes empresas son dueñas del $60,1 \%$ de la superficie con pino radiata y eucaliptos, las medianas empresas del 6,5\%, los medianos propietarios de un $11,2 \%$ y los pequeños propietarios de un $22,2 \%$ (Grosse y Rosselot, 2016).

Las exportaciones forestales desde el año 1980 se han multiplicado por 10 en casi 40 años, pasando de US\$ 500 mil a US\$ 6.838 millones en 2018 (INFOR, 2018), sin embargo, estas exportaciones corresponden a las grandes empresas, las exportaciones directas de pequeños propietarios son prácticamente inexistentes. Este escenario de desigualdad en el ingreso representa la evidencia económica y financiera del impacto de las brechas tecnológicas presentes en el sector forestal (INFOR, 2013).

En la cadena productiva del sector forestal se aprecian diversas brechas entre la pyme silvícola, representada por pequeños y medianos propietarios forestales, y el sector empresarial forestal industrial y exportador, lo cual requiere un trabajo efectivo para disminuirlas.

Los alcances de estos propietarios en la cadena del negocio forestal se limitan probablemente a la venta de su bosque a un intermediario a un precio estancado en el tiempo que lo fijan las grandes empresas del sector.

El Instituto Forestal, en el marco del proyecto INNOVA - BIO BIO "Agente de difusión y extensión tecnológica para pymes y propietarios forestales de la Región del Bio Bio", realizó una serie de actividades orientadas a la identificación y disminución de brechas tecnológicas en el segmento de pequeños productores de la región durante los años 2013 a 2016.

En este contexto se presentan los resultados de línea base en relación al grado de conocimiento y adopción de tecnologías en el ámbito del establecimiento y manejo de plantaciones forestales en pequeños propietarios de la Provincia de Arauco. De esta forma se busca dimensionar en cifras las brechas tecnológicas para procurar acciones tendientes a su progresiva reducción en el tiempo. 


\section{OBJETIVOS}

Dimensionar cualitativa y cuantitativamente el grado de conocimiento de pequeños propietarios en el establecimiento y manejo de plantaciones forestales en la Provincia de Arauco, Región del Bio Bio, Chile.

\section{MATERIAL Y MÉTODO}

El área de trabajo se estableció en la Provincia de Arauco, Región del Bio Bio, en las Comunas de Cañete, Los Álamos y Lebu. El estudio se realizó entre los años 2015 y 2016.

La Provincia de Arauco se emplaza en el sector sur poniente de la Región del Bio Bio, entre los paralelos $37^{\circ} 10^{\prime}$ y $38^{\circ} 34^{\prime}$ latitud sur y los meridianos $73^{\circ} 00^{\prime}$ y $73^{\circ} 41^{\prime}$ longitud oeste.

El sector de estudio presenta un clima templado infratermal homotérmico, mediterráneo húmedo de litoral, el régimen térmico se caracteriza por temperaturas que varían, en promedio entre una máxima de enero de $19,9^{\circ} \mathrm{C}$ y una mínima de julio de $6,2^{\circ} \mathrm{C}$. El período libre de heladas es de 320 días, con un promedio de 2 heladas por año. El régimen hídrico muestra una precipitación media anual de $1.261 \mathrm{~mm}$, un déficit hídrico de $411 \mathrm{~mm}$ y un período seco de 4 meses. La fuerte influencia marina determina una atenuación del régimen térmico con un verano fresco e invierno benigno (Agrimed-UdeChile, 2017).

En el marco del proyecto se crearon grupos de trabajo que se configuraron a partir de la información entregada por los programas permanentes de apoyo rural presentes en el territorio. Para el caso de las comunas en estudio se tiene al Programa de Desarrollo Local (PRODESAL) y el Programa de Desarrollo Territorial Indígena (PDTI). La condición exigida a las personas apoyadas por estos programas es que sean usuarios de INDAP conforme a su definición en la ley №18.910, de esta forma se asegura por parte del estudio la condición de pequeño propietario que es el sector objetivo. La presencia permanente de estos programas, algunos con varios años de funcionamiento, facilita el acceso a las personas en un contexto de trabajo colaborativo y de apoyo que permite una apertura al propietario, su predio y sus inquietudes.

Los criterios definidos para la selección de las personas fue que en sus predios tuvieran una plantación forestal de al menos 0,5 ha de cualquier edad y/o existiera en sus predios un terreno de aptitud forestal para establecer una plantación forestal con especies exóticas.

Bajo este esquema se definieron tres grupos de trabajo en el territorio, dos en la comuna de Cañete (64 personas) y uno considerando las comunas de Los Álamos y Lebu (27 personas). La división en estos grupos se define por la restricción logística y el número adecuado de personas en sala para las capacitaciones.

La herramienta elaborada por el equipo de trabajo para la recopilación de datos para definir la línea base fue una encuesta semiestructurada relativa a las técnicas empleadas para el establecimiento y para el manejo de las plantaciones forestales. La entrevista fue presencial y se realizó en el predio de cada propietario. La encuesta fue ejecutada por dos profesionales, en la cual uno registraba y el segundo conducía la conversación.

Las preguntas estaban enfocadas a cómo ejecutó sus plantaciones forestales y el manejo de estas cuando correspondiese. Las preguntas estaban dirigidas a identificar las actividades definidas en los protocolos de plantaciones ampliamente aceptados en el ámbito académico y productivo (García et al., 2002a; Sotomayor et al., 2002). En forma paralela se visitaron las plantaciones forestales cuando correspondiera, se realizaron mediciones dasométricas y se tomaron registros fotográficos.

Las encuestas fueron procesadas en planillas electrónicas y sus datos fueron procesados, graficados y analizados en términos porcentuales con sus estadígrafos descriptivos. 


\section{RESULTADOS}

\section{Caracterización Socioeconómica Grupo Objetivo}

En la Figura $\mathrm{N}^{\circ} 1$ se muestran los datos socioeconómicos de los pequeños propietarios rurales de la Provincia de Arauco con los cuales se trabajó, pertenecientes a la agricultura familiar campesina y reconocidos como usuarios de INDAP, de acuerdo con la clasificación señalada en la ley 18.910.

Se aprecia que el rango etario está dominado por adultos y adultos mayores, con una representatividad de género prácticamente igualitaria. El grupo de estudio se caracteriza por una baja escolaridad, representada por un $63 \%$ que solo tiene formación básica incompleta con un máximo de 8 años de escuela básica rural. Otro rasgo distintivo de este grupo es la presencia mayoritaria de la etnia mapuche, la cual se representa en gran parte del territorio de la Provincia de Arauco.
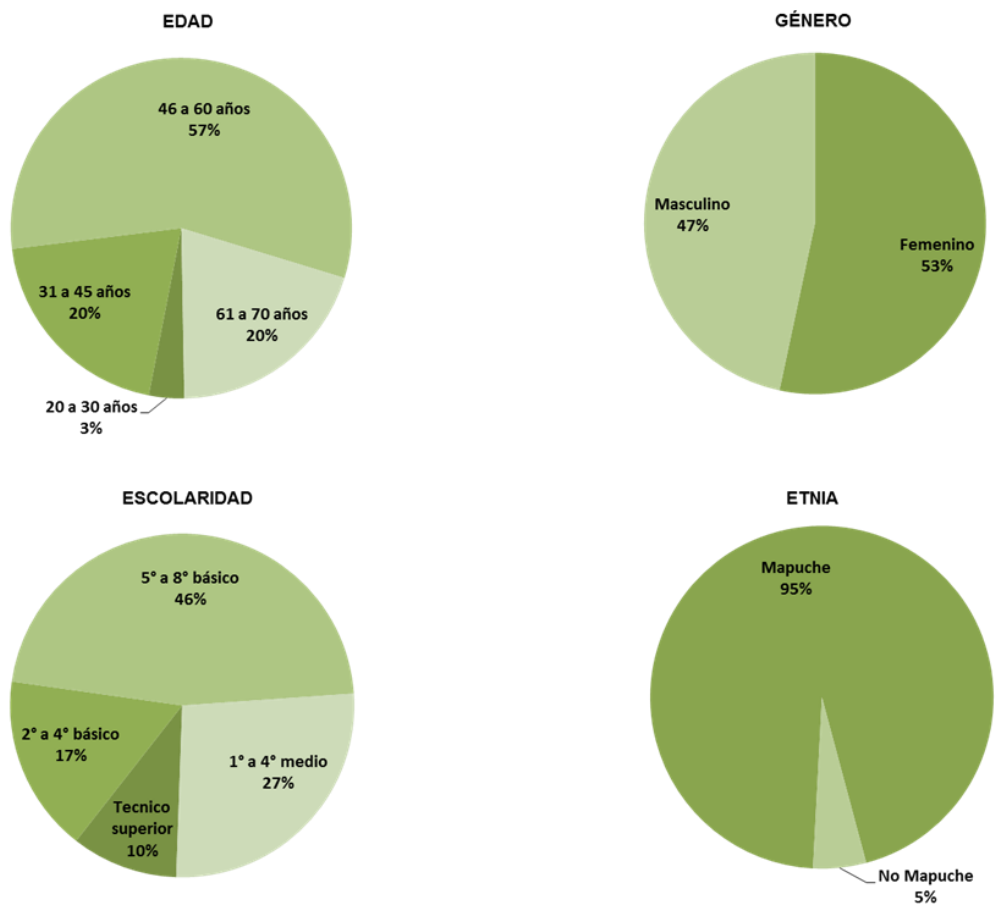

Figura $\mathrm{N}^{\circ} 1$

ANTECEDENTES SOCIOECONOMICOS DE PEQUEÑOS PROPIETARIOS DEL ESTUDIO

En relación a los antecedentes prediales, en la Figura $\mathrm{N}^{\circ} 2$ se detallan los principales aspectos de sus terrenos, en especial el tamaño predial, el cual mayoritariamente se clasifica en propiedades menores a 20 ha de superficie. Proporcional a este tamaño predial se clasifican las plantaciones forestales existentes a la fecha del estudio en estas propiedades, en donde la mayor frecuencia encontrada se ubica en el rango de 1 a 5 ha de plantaciones forestales con especies exóticas. 
TAMAÑO PREDIAL

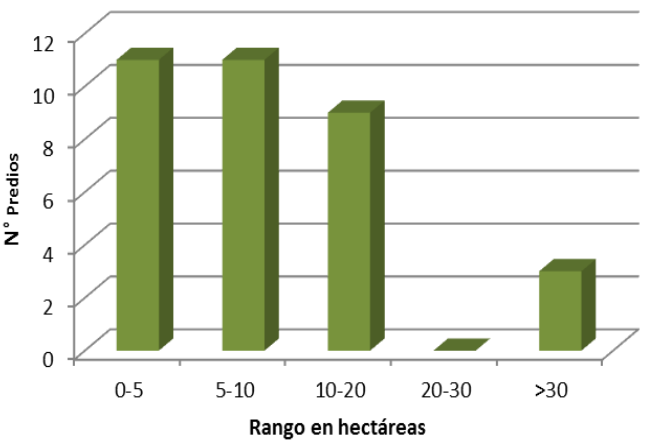

TAMAÑO PLANTACIONES FORESTALES

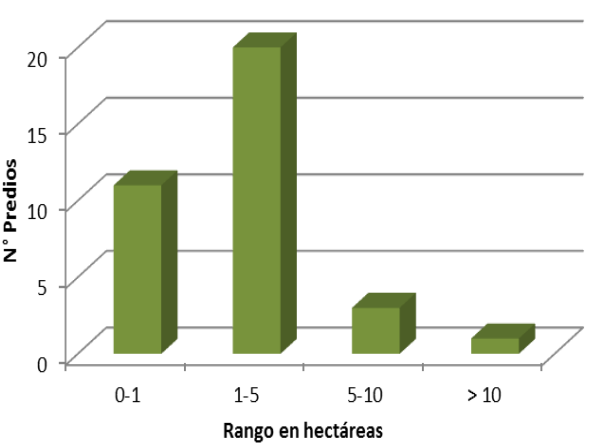

Figura $\mathrm{N}^{\circ} 2$

ANTECEDENTES PREDIALES DE PEQUEÑOS PROPIETARIOS DEL ESTUDIO

\section{Gestión de Plantación}

La Figura $\mathrm{N}^{\circ} 3$ muestra algunos indicadores referentes a la gestión de plantación de pequeños propietarios de la Provincia de Arauco, destacándose el nulo apoyo y escasas herramientas accesibles para garantizar el éxito de una plantación forestal.

Se observa que casi la totalidad de estos propietarios asumió la faena en forma directa, vale decir ellos mismos fueron los ejecutores de la plantación, sin embargo, solo un $26 \%$ de ellos presentaba algún grado de capacitación al respecto, relacionado fundamentalmente con la participación en faenas de plantación de grandes empresas.

Esta gestión directa se traduce en la ocupación del tiempo propio y de sus limitados conocimientos para ejecutar las distintas faenas que comprende la plantación.

La gestión directa comprende todo el proceso de plantación desde la compra de las plantas hasta la faena de plantación en terreno y las etapas que esta involucra.

Además, un escaso $7 \%$ de ellos utilizó los mecanismos de fomento establecidos en el DL 701 sobre incentivos a la forestación que, hasta el año 2012, financiaba el $90 \%$ de los costos de forestación de pequeños propietarios.

Con estos indicadores se establece un escenario de alta incertidumbre para el desarrollo y éxito de una plantación forestal.

Se trata de pequeños propietarios que asumen un proyecto de largo plazo de forma directa, que no lo conocen suficientemente ni han sido capacitados y, que finalmente, lo ejecutan con recursos propios. 

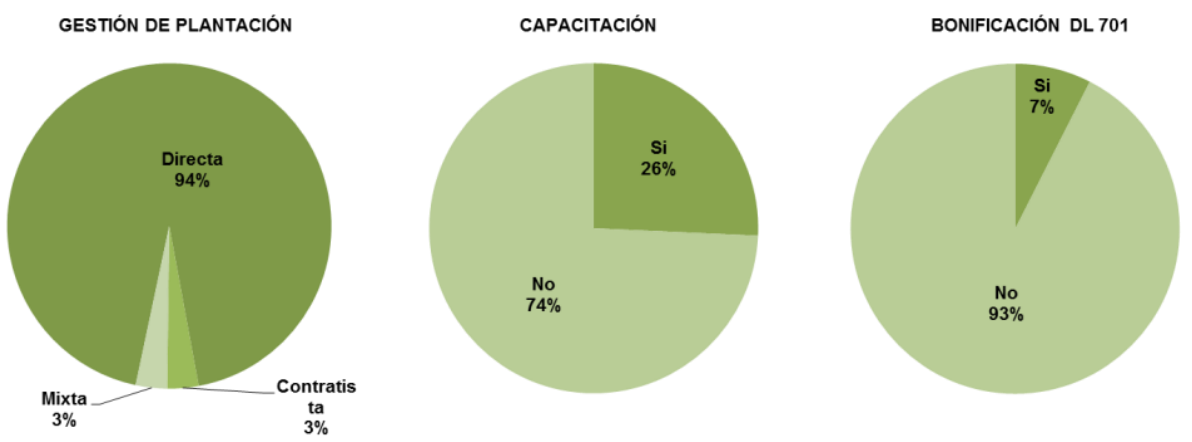

Figura $\mathbf{N}^{\circ} 3$

INDICADORES DE GESTION DE PLANTACIÓN DE PEQUEÑOS PROPIETARIOS DEL ESTUDIO

\section{Establecimiento de Plantaciones}

En la Figura $\mathrm{N}^{\circ} 4$ se observa la preferencia por la especie Eucalyptus globulus, la cual ocupa mayoritariamente los territorios destinados a plantación, dada la buena respuesta que tiene esta especie en la Provincia de Arauco por las favorables condiciones agroclimáticas que determinan rápidos crecimientos junto a la característica de rebrote que presenta después de la corta. Por su parte el pino radiata tiene un $13 \%$ de representatividad en este grupo de pequeños propietarios.

En la misma figura, se observa el gráfico de preparación de suelo en el cual un altísimo $77 \%$ responde que no realiza labores de preparación de suelo antes de realizar una plantación forestal, entendiendo por esta cualquier labor de cultivo del suelo para facilitar el prendimiento de la plantación, limitándose solamente a realizar una casilla manual para la instalación de la planta, que no cumple cabalmente con la remoción de suelo recomendada técnicamente (García et al., 2002b).
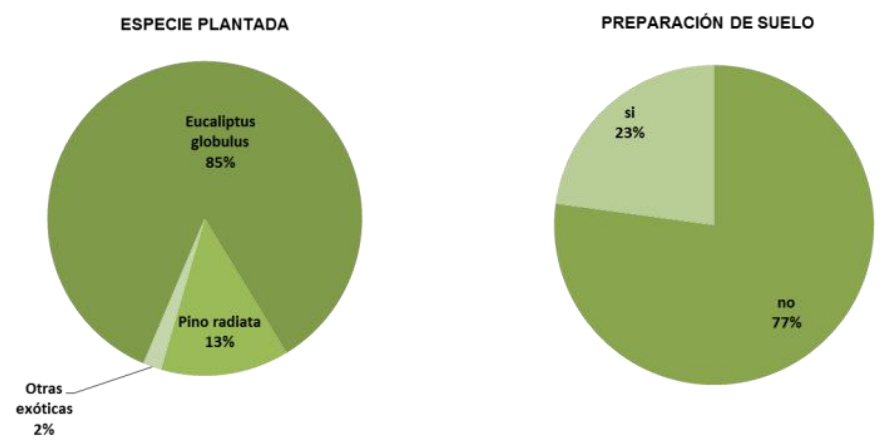

Figura $N^{\circ} 4$ ESPECIE FORESTAL PLANTADA Y PREPARACIÓN DE SUELO EN LA PLANTACIÓN

Respecto de la faena de roce previa a la plantación, la mayoría contesta que sí realizan esta faena, que se ejecuta en forma manual, y que los desechos generados son mayoritariamente quemados $(71 \%)$, práctica habitual entre los agricultores, pero que lamentablemente provoca un daño en las capas superiores del suelo, disminuyendo la capacidad nutritiva de este para soportar vegetación, además del serio riesgo de provocar incendios (Figura $N^{\circ} 5$ ). 

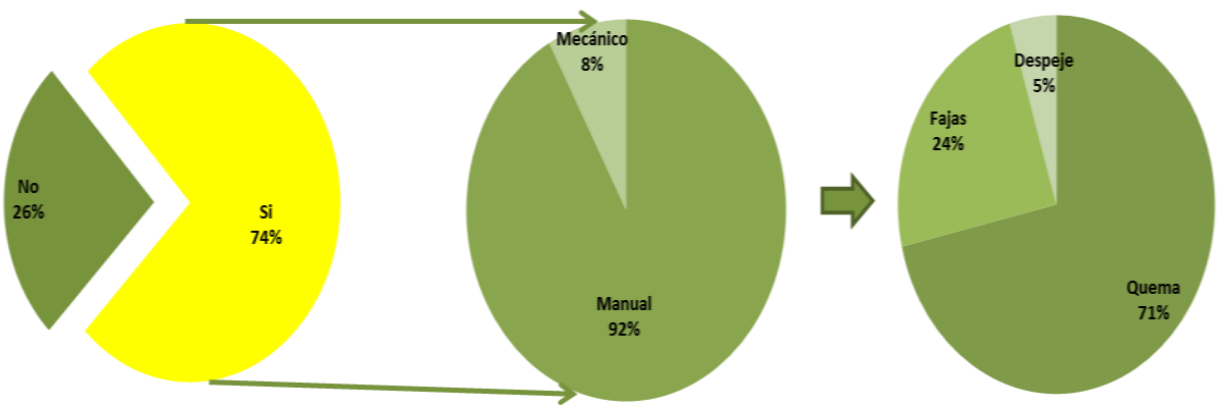

Figura $\mathrm{N}^{\circ} 5$

ANTECEDENTES DE FAENA DE ROCE Y TRATAMIENTO DE DESECHOS

En cuanto al origen de las plantas utilizadas y las decisiones de establecimiento, los antecedentes indican que las plantas proviene mayoritariamente de viveros comerciales, los cuales corresponden a pequeños y medianos viveros generalmente ubicados en las cercanías de los predios y que presentan una calidad de planta menor, un $11 \%$ establece su propio vivero para la generación de las plantas y un $8 \%$ lo obtiene de regeneración de un bosque cercano, trasplantando estas plantas hasta el lugar de establecimiento en sus predios (Figura $\mathrm{N}^{\circ} 6$ ).

gestión

Cabe destacar la escasa presencia institucional y de las empresas en el apoyo de esta

El tipo de planta utilizada es en un $65 \%$ proveniente de cultivo en bandejas (speedling trays) y un importante $27 \%$ emplea plantas a raíz desnuda.

La temporada de plantación está bien definida en la temporada de invierno, no obstante, un $21 \%$ realiza la plantación después de septiembre lo cual se identifica como una plantación tardía que dificulta un mejor establecimiento de esta.

Al observar la densidad de plantación definida por los propietarios se observa una variada combinación de medidas, predominando el espaciamiento de $2 \times 3 \mathrm{~m}$, que es ampliamente aceptado como adecuado para una plantación forestal pura.

No obstante, cerca de un $30 \%$ de los pequeños propietarios practica una alta densidad de plantación, que implica un mayor costo de establecimiento y un menor desarrollo global de la plantación forestal. 


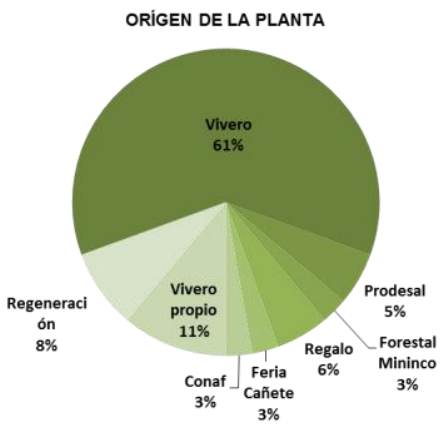

TEMPORADA PLANTACIÓN

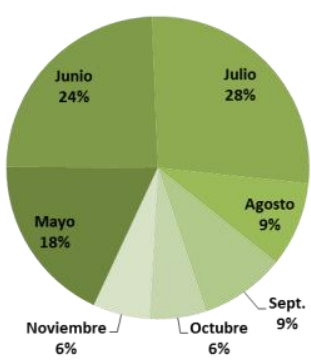

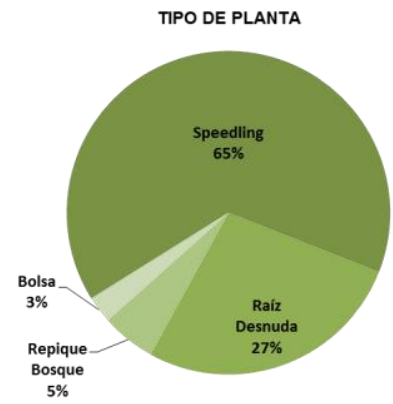

DISTANCIAMIENTO PLANTACIÓN

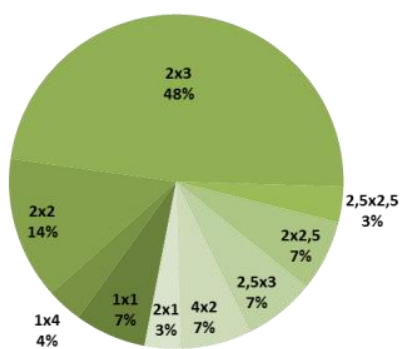

Figura $\mathrm{N}^{\circ} 6$

ANTECEDENTES DE LA PLANTA Y LABORES DE PLANTACIÓN UTILIZADA POR PEQUEÑOS PROPIETARIOS

Consultados por la faena de fertilización en la plantación forestal, la mayoría de los pequeños propietarios respondieron afirmativamente $(67 \%)$, no obstante, un alto $33 \%$ no realiza fertilización.

Respecto del fertilizante utilizado, existe una gran variedad de distintos productos, un porcentaje predominante $(31 \%)$ utiliza el llamado fertilizante mezcla forestal que es una formulación comercial que se vende en el mercado, no obstante, un porcentaje similar responde que no sabe que fertilizante utilizó (Figura $\mathrm{N}^{\circ} 7$ ).

Tomando como base el llamado fertilizante mezcla forestal, se indagó respecto de la dosis utilizada al momento de plantar, obteniendo nuevamente una amplia variedad de respuestas que indican que una cuarta parte de los propietarios no sabe qué dosis aplicó y el resto presenta variaciones de hasta un $300 \%$ en la cantidad de fertilizante. La dosis recomendada para esta mezcla es aproximadamente $100 \mathrm{~g}$ por planta (García et al., 2002b), y para este caso de análisis solo la opción vaso de yogurt se acerca a lo recomendado. 


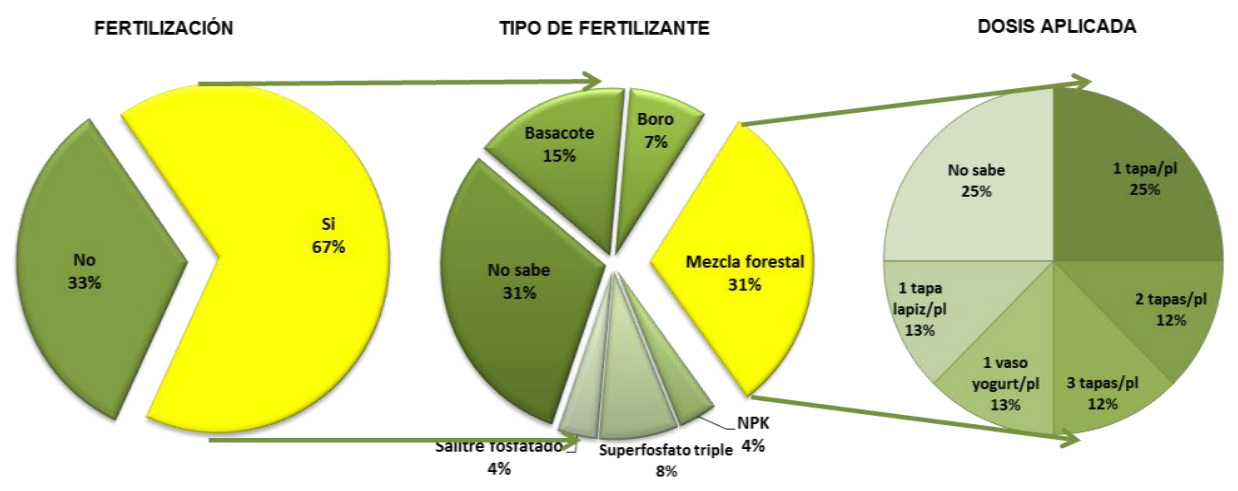

Figura $\mathrm{N}^{\circ} 7$

ANTECEDENTES DE FAENA DE FERTILIZACIÓN UTILIZADA EN PLANTACIÓN FORESTAL

Posteriormente se analizó la faena de control de maleza en el establecimiento de la plantación forestal (Figura $\mathrm{N}^{\circ} 8$ ). Solo un $27 \%$ de los propietarios realizó un control de maleza previo a la plantación, el cual se ejecutó mayoritariamente en forma manual.

Es importante recordar que el control de maleza se considera como una de las faenas prioritarias para el éxito de una plantación forestal (Álvarez et al., 2004).

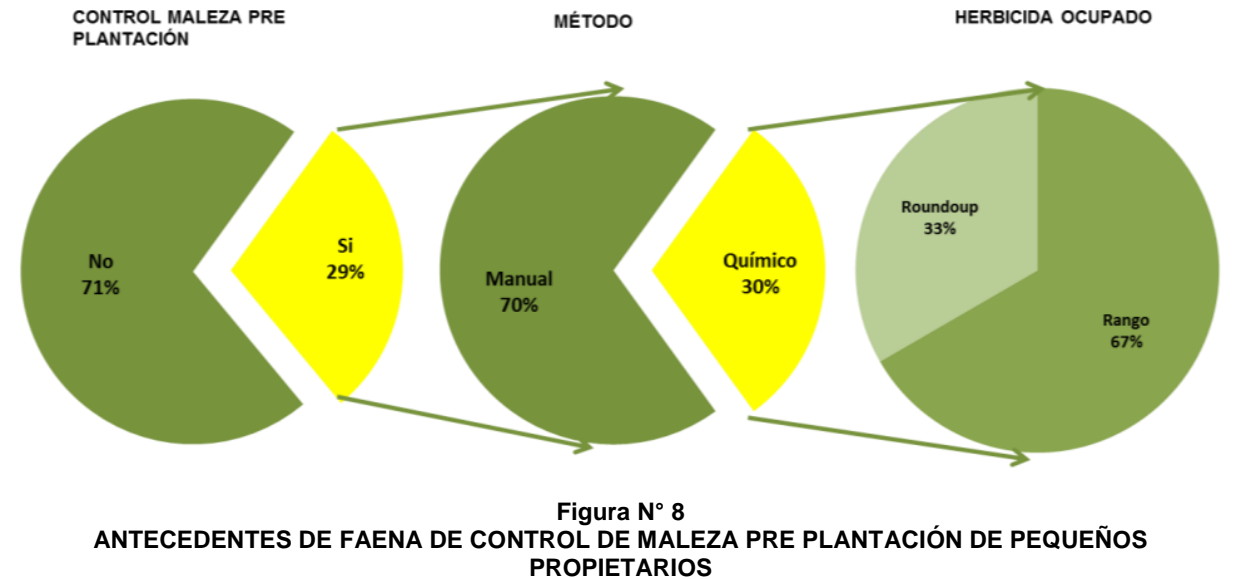

Tanto o más importante es el control de maleza posterior a la plantación, momento en que se establece la competencia directa de la vegetación herbácea con la planta recientemente instalada.

En la Figura $\mathrm{N}^{\circ} 9$ se observa que solo un $18 \%$ de los propietarios ejecutó un control de maleza después de instalada la planta, el cual se realizó principalmente en forma manual y especialmente durante el primer año de la plantación, solo un $29 \%$ repitió este control durante el segundo año. 


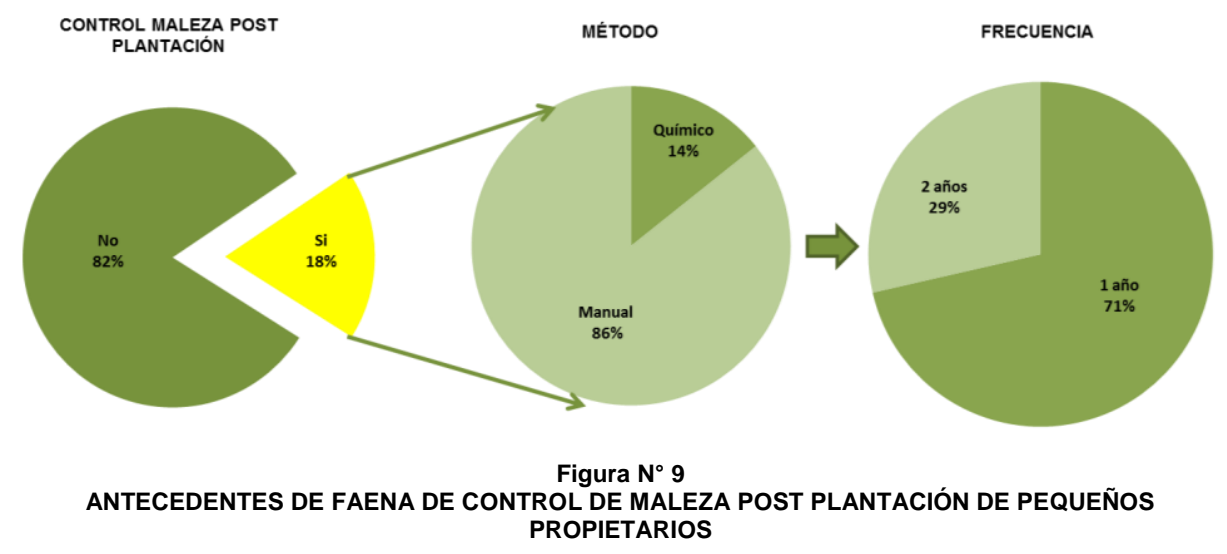

Una vez realizadas las consultas por las distintas actividades asociadas al establecimiento de una plantación, se les consultó por la actividad de replante. Se observa en la Figura $\mathrm{N}^{\circ} 10$, que una amplia mayoría realizó una labor de replante en su predio (75\%). La actividad de replante, consiste en el reemplazo de aquellas plantas que se murieron durante el verano y no fueron capaces de soportar la sequía, el calor y las condiciones de suelo adversas que les propone el establecimiento en terreno. Respecto de la intensidad de este replante, prácticamente un cuarto de los propietarios realizó un replante superior al $30 \%$ respecto del número de plantas iniciales.

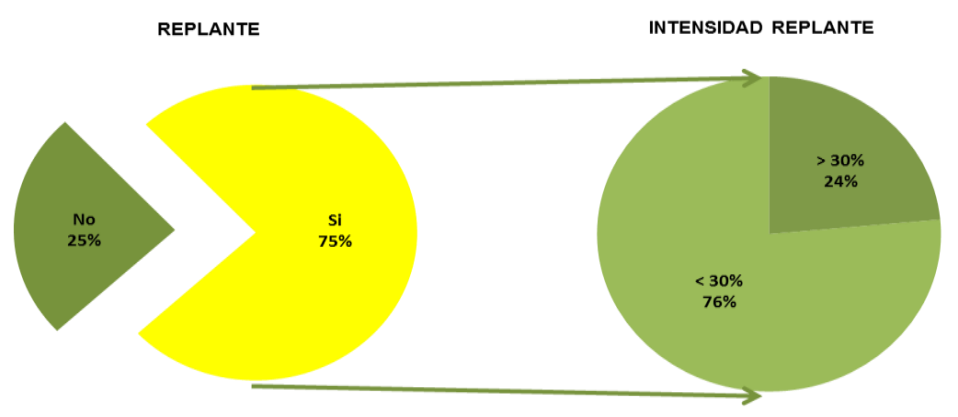

Figura $\mathrm{N}^{\circ} 10$

DATOS DE FAENA DE REPLANTE DE PEQUEÑOS PROPIETARIOS

\section{Manejo de Plantaciones}

Para la mayoría de los propietarios el manejo aparece como una labor poco relevante en el desarrollo de una plantación y aquellos que consideran que tiene cierta incidencia en la calidad de los productos realizan algún tipo de faena, tanto en pino como en eucalipto. No obstante, al consultarles por labores más específicas, como raleo en las plantaciones se pino, solo un $14 \%$ respondió haberlo realizado en plantaciones factibles de ralear. La amplia mayoría no efectuó raleo en su plantación de pino. En cuanto al eucalipto, las actividades de manejo se refieren al manejo de rebrote de esta especie después de la corta. 

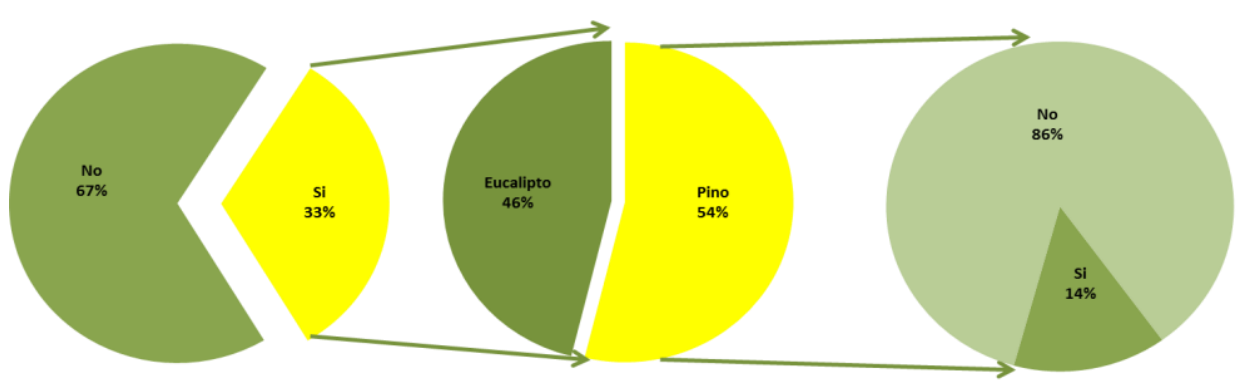

Figura $\mathrm{N}^{\circ} 11$

\section{ANTECEDENTES DE MANEJO DE PLANTACIONES DE PEQUEÑOS PROPIETARIOS}

En lo que se refiere a la faena de poda en pino radiata (Figura $N^{\circ} 12$ ), un $43 \%$ de los propietarios que realizaron manejo declaró haber realizado una poda en su plantación. Consultados por los objetivos de poda, un cuarto de ellos no sabe específicamente el objetivo y una proporción idéntica declara que es para la cosecha, solo la mitad de ellos se acerca al objetivo de la poda en pino radiata.
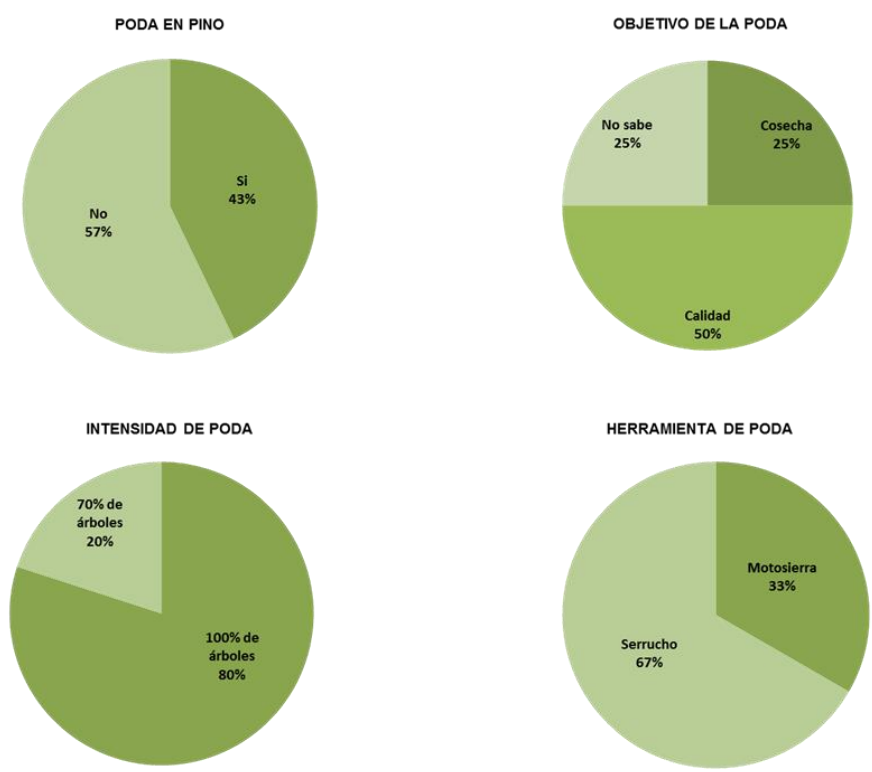

Figura $\mathrm{N}^{\circ} 12$

ANTECEDENTES DE FAENA DE PODA EN PINO RADIATA DE PEQUEÑOS PROPIETARIOS 
No obstante, respecto de la intensidad de poda, un porcentaje mayoritario realizó poda al $100 \%$ de los árboles presentes en la plantación, evidenciando un escaso conocimiento de la faena y su propósito. En en relación a la herramienta de trabajo, un tercio de ellos lo realiza con una motosierra, lo que es técnicamente incorrecto y operacionalmente riesgoso. Además, de acuerdo a lo observado en terreno, el total de las podas realizadas estaban equivocadas en cuanto a la oportunidad de la intervención, siendo estas muy tempranas o muy tardías. Se aprecia una desconexión con el propósito de la poda, su frecuencia y la cantidad necesaria de intervenciones.

\section{DISCUSION Y CONCLUSIONES}

Los resultados entregados en este informe reflejan en forma detallada cómo la suma de factores explica las diferencias de calidad y productividad entre las plantaciones forestales de pequeños propietarios y aquellas pertenecientes a las empresas.

La falta de capacitación y de conocimientos específicos del rubro forestal son materias evidentes en esta radiografía extraída en estos grupos de trabajo, y la sumatoria de factores va produciendo un efecto negativo en la productividad de las plantaciones, ya que decisiones equivocadas por falta de conocimiento derivan en una pérdida económica irrecuperable en el periodo de rotación de los bosques. Uno de los aspectos llamativos y preocupantes es que esto ocurre en la Región del Bio Bio, que concentra casi el $40 \%$ de las plantaciones forestales del país, pero es el reflejo de las asimetrías tecnológicas y de conocimiento que existen entre las grandes empresas y los pequeños propietarios.

Dentro de las cifras más elocuentes destaca lo relativo a la gestión de plantación, un $94 \%$ de los pequeños propietarios lo hace por gestión directa en circunstancias que el $75 \%$ declara no haber recibido capacitación alguna. Inician así un proyecto de largo plazo, con los limitados recursos propios y con serias carencias tecnológicas, situación que conduce a plantaciones de baja calidad y escasa productividad. Evidencia de esto son los altos porcentajes de replante, lo que indica una plantación deficiente desde su origen.

La actual situación hace temer que estas brechas se mantengan o amplíen mientras los pequeños propietarios no cuenten con los conocimientos técnicos suficientes que les permitan una autosuficiencia en su gestión del recurso. Pino y eucalipto son las especies que cuentan con mayor respaldo tecnológico en términos de silvicultura, manejo y mejoramiento genético y la silvicultura intensiva aplicada en ellas genera una alta productividad de las plantaciones. No obstante, estas técnicas no llegan a los pequeños propietarios, dados sus escasos recursos y la insuficiente transferencia y capacitación a la que pueden acceder.

La falta de manejo en las plantaciones de pino radiata o el manejo inadecuado y fuera de oportunidad representa un sombrío panorama para el futuro del recurso creado por este segmento de propietarios y para las expectativas económicas en torno a este.

Ciertamente los pequeños propietarios no cifran su ingreso económico en las plantaciones forestales, que son una opción de largo plazo en los suelos más pobres de sus predios, no obstante, la inversión y los esfuerzos son importantes. Con algo más de tecnología y capacitación estos esfuerzos debieran llevar a mejores resultados económicos y a una adecuada protección de suelos, elementos gravitantes en los predios pequeños y medianos propietarios.

Se concluye que los pequeños propietarios de la Provincia de Arauco, Región del Bio Bio, principal región del país en materia de desarrollo en plantaciones forestales, después de más de 50 años de desarrollo tienen un escaso conocimiento respecto de técnicas de establecimiento de plantaciones forestales y muy escaso en lo referente a manejo de estas, situación que explica en gran parte las brechas existentes. Urge así una intensificación de los programas de transferencia y capacitación de los organismos del Agro. 


\section{REFERENCIAS}

AGRIMED - U. de CHILE, 2017. Atlas Agroclimático de Chile. Estado Actual y Tendencias del Clima. Tomo IV: Regiones del Bio Bio y la Araucanía. Universidad de Chile-FIA. Santiago, Chile. 136p.

Álvarez, J.; Venegas, R. y Pérez, C., 2004. Impacto de la Duración y Geometría del Control de Malezas en la Productividad de Plantaciones de Pinus radiata D. Don en Cinco Ecosistemas del Sur de Chile. Revista Bosque 25 (2):57 -67.

García, E.; Sotomayor, A.; Silva, S. y Valdebenito, G., 2002a. Establecimiento de Plantaciones Forestales. Eucalyptus sp. Documento de Divulgación No 18. INFOR-FDI. Santiago, Chile. 30p.

García, E.; Sotomayor, A.; Silva, S. y Valdebenito, G., 2002b. Establecimiento de Plantaciones Forestales. Pinus radiata, Pinus ponderosa y Pseudotsuga menziesii. Documento de Divulgación № 17 . INFOR-FDI. Santiago, Chile. 33p.

Grosse, H. y Rosselot, F., 2016. La Potencialidad de Nuevas Plantaciones Forestales en Chile. Revista Ciencia e Investigación Forestal 22 (1):77-89.

INFOR, 2013. Anuario Forestal 2013. Boletín Estadístico № 140. Instituto Forestal. Santiago, Chile. 148p.

INFOR, 2018. Anuario Forestal 2018. Boletín Estadístico № 163. Instituto Forestal. Santiago, Chile. 177p.

Sotomayor, A.; Helmke, E. y García, E. 2002. Manejo y Mantención de Plantaciones forestales. Pinus radiata y Eucalyptus sp. Documento de Divulgación № 23. INFOR-FDI. Santiago, Chile. 56 p. 
